\title{
Coraliomela brunnea brunnea: morfologia do adulto e sinonímias (Coleoptera, Chrysomelidae)
}

\author{
Carla S. Jung ${ }^{1} \&$ Marcela L. Monné ${ }^{1,2}$ \\ 1. Museu de Zoologia, Universidade de São Paulo, Caixa Postal 42494, 04218-970 São Paulo, SP, Brasil. \\ 2. Bolsista da FAPESP.
}

\begin{abstract}
Coraliomela brunnea brunnea: adult morphology and synonyms (Coleoptera, Chrysomelidae). The adult of Coraliomela brunnea brunnea (Thunberg, 1821) is redescribed and illustrated including new morphological characters. Coraliomela brunnea collaris (Guérin-Méneville, 1840) and C. brunnea nigripes (Guérin-Méneville, 1840) are considered synonyms of $C$. brunnea brunnea.
\end{abstract}

KEYWORDS. Alurnini, Coraliomela, Hispinae.

RESUMO. O adulto de Coraliomela brunnea brunnea (Thunberg, 1821) é redescrito e ilustrado incluindo novos caracteres morfológicos. Coraliomela brunnea collaris (Guérin-Méneville, 1840) e C. brunnea nigripes (Guérin-Méneville, 1840) são considerados sinônimos de C. brunnea brunnea.

PALAVRAS-CHAVE. Alurnini, Coraliomela, Hispinae.

O gênero Coraliomela Jacobson, 1899 apresenta atualmente quatro espécies: $C$. brunnea (Thunberg, 1821), C. thoracica (Perty, 1832), C. aenoplagiata (Lucas, 1857) e C. quadrimaculata (Guérin-Méneville, 1840), que ocorrem no Brasil, Bolívia, Paraguai e Argentina (FISHER, 1935; Monrós \& Viana, 1947; Staines, 2002).

ThunBerg (1821) descreveu Alurnus brunneus do Brasil. JACOBSON (1899) propôs o subgênero Coraliomela sob Mecistomela Jacobson, 1899. Fisher (1935) elevou o status subgenérico de Coraliomela para o nível de gênero, sinonimizou Mecistomela (Coraliomela) corallina (Vigors, 1826) com Alurnus brunneus Thunberg, 1821, transferiu-a para Coraliomela e designou-a como espécie-tipo; considerou, ainda, quatro subespécies sob C. brunnea: C. brunnea nigripes (Guérin-Méneville, 1840), C. brunnea vigorsi (GuérinMéneville, 1840), C. brunnea thoracica (Perty, 1832) e $C$. brunnea nigerrima (Jacobson, 1899). BLACKWELDER (1946) arrolou, em Coraliomela brunnea, as quatro subespécies consideradas por FisCHER (1935). MONRÓs \& Viana (1947), ao revisarem as espécies de Hispinae que ocorrem na Argentina, elevaram C. brunnea thoracica para C. thoracica e consideraram duas subespécies em C. brunnea: C. brunnea collaris (GuérinMéneville, 1840), que foi revalidada, e C. brunnea nigripes.

Neste trabalho, Coraliomela brunnea collaris e C. brunnea nigripes são consideradas sinônimas de $C$. brunnea brunnea. Coraliomela brunnea vigorsi e $C$. brunnea nigerrima são mantidas no status original devido à falta de material para estudo.

\section{MATERIAL E MÉTODOS}

$\mathrm{O}$ material examinado pertence às seguintes instituições: Departamento de Zoologia, Universidade Federal do Paraná, Curitiba (DZUP); Museu de Zoologia, Universidade de São Paulo, São Paulo (MZSP) e Museu
Nacional, Universidade Federal do Rio de Janeiro, Rio de Janeiro (MNRJ). Para a maioria das estruturas empregouse a terminologia tradicional em Chrysomelidae e/ou Coleoptera. Para venação alar seguiu-se JoLIVET (1954); para o metendosternito Crowson $(1938,1944)$; para a terminália masculina LindRoth \& PALMÉN (1970) e MANN (1985); para a terminália feminina TANNER (1927) e KASAP $\&$ CROWSON (1985). Com base na morfologia externa não foi possível sexar os exemplares.

\section{Coraliomela brunnea brunnea (Thunberg, 1821)}

$$
\text { (Figs. 1-17) }
$$

Alurnus brunneus THUNBerg, 1821:200; JACOBSON, 1924:241. Alurnus corallinus VIgORS, 1826:240; BONDAR, 1915a:23; $1915 \mathrm{~b}: 441$

Alurnus corallinus var. A. BALY, 1858:26.

Alurnus coccineus Guérin-Méneville, 1840:331.

Alurnus sanguineus Guérin-MénEvilLe, 1840:331.

Mecistomela (Coraliomela) corallina; JACOBSON, 1899:254; BERG, 1900:255; WeIse, 1910:105; 1911a:14; 1911b:20; Bruch, 1914:375; Bondar, 1922:48; 1931:136; Moreira, 1921:471; 1929:79, 82; LiMA, 1923:190; 1927:189; 1936:326; RonNA, 1928:37; FISCHER, 1935:280

Mecistomela (C.) corallina var. sanguinea; JACOBSON, 1899:255. Mecistomela $(C$.) thoracica var. phenax JACOBSON, 1899:254.

Coraliomela brunnea; Fischer, 1935:280; BosQ, 1943:40; BlackWelder, 1946:722; Monrós \& Viana, 1947:177; Lima, 1955:204; Staines, 1991:247.

Alurnus collaris GUÉrin-MénevilLe, 1840:331; FisCher, 1935:280. Syn. nov.

Mecistomela (Coraliomela) coralina var. collaris; JACOBSON, 1899:254; WeIse, 1911a:14; 1911b:20

Coraliomela brunnea collaris; MonRós \& Viana, 1947:179.

Alurnus nigripes Guérin-MÉneville, 1840:331; Baly, 1858:27. Syn. nov.

Mecistomela nigripes; SPAETH, 1937:144.

Mecistomela (Coraliomela) nigripes; JACOBSON, 1899:253; BERG, 1900:254; WeIse, 1910:106; 1911a:14; 1911b:21; BRUCH, 1914:376.

Coraliomela brunnea nigripes; FISCHER, 1935:282; BLACKWELDER, 1946:722; Monrós \& Viana, 1947:179. 
Corpo robusto e largo, sem dimorfismo sexual. Região dorsal do corpo vermelha exceto antenas e escutelo, pretos; pronoto totalmente vermelho ou com faixa longitudinal mediana preta. Região ventral preta; pernas unicolores, pretas ou com fêmures vermelhos.

Cabeça quase tão larga quando longa e com pilosidade curta, esbranquiçada e esparsa. Vértice com pontos densos, rasos e irregulares, superfície microesculturada. Fronte curta e oblíqua. Olhos ovais e finamente facetados. Base das antenas, escapo e pedicelo com pontos finos, esparsos, rasos e densos. Labro subretangular, com pontos finos e densos, pêlos curtos e castanhos. Mandíbulas (Figs. 1, 2) triangulares, distintamente curvas, com margem interna sem dentes e ápice projetado, arredondado. Maxila (Fig. 3) com gálea arredondada e pilosidade curta na margem; palpos maxilares com pêlos curtos e esbranquiçados, artículo basal curto, cilíndrico, com 1/3 do comprimento do apical; II e III cônicos e subiguais, artículo apical cerca de duas vezes mais longo que o anterior e arredondado no ápice. Lábio (Fig. 4) com mento estreito e transverso; artículo I dos palpos labiais curto e transverso; II e III com comprimento subigual e cerca do dobro do comprimento do I; III arredondado no ápice. Antenas com 11 segmentos, curtas, alcançam a margem posterior do protórax e com pêlos curtos, esparsos e pouco aparentes; pedicelo curto e transverso; antenômero III 1/3 mais longo que o IV; IV-VII subiguais em comprimento; VIII$\mathrm{XI}$ subiguais, com cerca da metade do comprimento do III.

Protórax transverso. Pronoto cerca do dobro do comprimento do prosterno, microesculturado, com pontos grossos e irregulares, exceto a faixa mediana longitudinal, lisa. Processo prosternal (Fig. 5) alargado para o ápice, maior largura subigual ao diâmetro de uma cavidade coxal e com a margem posterior sub-reta; cavidades cotilóides anteriores angulosas aos lados e fechadas atrás. Proendosternito (Fig. 6) pigmentado, mais longo que largo, com projeções alargadas na base, arredondadas no ápice, distantes entre si e subparalelas. Processo mesosternal (Fig. 7) tão largo quanto o diâmetro de uma cavidade mesocoxal; cavidades cotilóides médias abertas lateralmente. Mesendosternito (Fig. 9) obliquamente dirigido para o mesepímero e fusionado no ápice à parede interna do mesmo. Metasterno aplanado, com rugas transversais e pontos finos rasos e esparsos. Metepisterno (Fig. 8) estreito na região mediana, com superfície microesculturada. Metendosternito (Fig. 10) com lâminas longas e largas em relação aos braços, separadas por chanfro inconspícuo; pedúnculo curto, bilobado; braços perpendiculares às lâminas e divergentes entre si; implantes dos tendões nulos. Escutelo subtriangular.

Élitros três vezes mais longo que largo, estreitado no quarto apical, com ápices arredondados e cerca de quatro vezes o comprimento do protórax; pontuação grossa, microesculturada, moderadamente ordenada em fileiras e superfície glabra, exceto o quarto apical dotado de cerdas curtas entre os pontos, esparsas e amareladas. Úmeros arredondados. Asas posteriores (Fig. 12) pelo menos duas vezes mais longas que largas; lobo anal moderadamente desenvolvido; Costa $(\mathrm{C})$ reduzida, restrita à região basal; Subcosta (Sc) alcança a metade basal da asa; célula da radial (cr) fechada com aspecto triangular; r-s evidente; árculo (arc) presente; s-m vestigial; Cubital $(\mathrm{Cu})$ bem desenvolvida, Média-Anterior (MA) vestigial, Plical (P) sem ramificações;
Empusal (E) unida à primeira anal (1A); transversa 2Aa, Célula anal $(\mathrm{Ca})$ e $2 \mathrm{Ab}$ presentes; Jugal (Ju) reduzida.

Pernas curtas e subiguais em comprimento; procoxa transversa e ligeiramente proeminente; mesocoxa arredondada. Trocanteres com tufo de pêlos amarelos na margem inferior. Fêmures subcilíndricos, ligeiramente engrossados na região mediana. Tíbias tão longas quanto os fêmures, algo alargadas para a extremidade, carenadas no bordo externo, com pilosidade preta densa no quarto apical. Tarsômero I subtriangular, 1/3 mais curto que o II; II-III subiguais em comprimento, bilobados, com bilobação mais evidente no III; IV reduzido e V ligeiramente arqueado, com um par de garras simples na extremidade. Empódio (Fig. 11) com região apical acuminada, reduzida, cerca de $1 / 3$ da basal e sem cerdas.

Esternitos I-V com pontuação fina, esparsa e pêlos curtos e esparsos. Esternitos I-IV estreitos e subiguais em comprimento. Esternito V cerca de 1/3 mais longo que o anterior e margem apical arredondada.

Genitália masculina. Lobo médio (Figs. 14, 15) alongado, esclerotizado, encurvado quando observado lateralmente; extremidade apical estreitada e arredondada; saco interno membranoso, com ducto ejaculatório estreito, longo, pelo menos tão longo quanto o comprimento do lobo médio. Tégmen (Fig. 13) em forma de forquilha, prende-se à região basal do lobo médio; projeção apical com cerca da metade do comprimento da região em forquilha.

Genitália feminina. Esternito VIII (Fig. 16) pouco esclerotinizado, com região apical curta, transversa e margem truncada; apódema cerca de três vezes mais longo que a região apical. Ovipositor (Fig. 17) curto, terço apical transverso, pigmentado, com palpos vaginais reduzidos e vulva plicada. Ducto espermatecal longo, distintamente enrolado e pigmentado; espermateca curva, região basal alargada e apical afilada.

Dimensões em mm. Comprimento total, 23,0-31,2; comprimento do protórax, 4,5-5,8; maior largura do protórax, 6,58,0; comprimento do élitro, 17,7-23,2; largura umeral, 9,1-11,5.

Discussão. Os sinônimos propostos basearam-se nas descrições originais e no material examinado. As diferenças entre as subespécies referem-se à coloração, tal como ocorre em Coraliomela brunnea nigripes, que apresenta os fêmures pretos e em Coraliomela brunnea collaris cujo pronoto é inteiramente vermelho. Nos exemplares examinados, tanto a coloração dos fêmures como a do pronoto varia consideravelmente independentemente da distribuição geográfica, ficando impossível de se manter as subespécies.

Coraliomela brunnea brunnea difere das demais espécies do gênero pelos élitros inteiramente vermelhos.

Distribuição geográfica. Brasil (Pará, Rondônia, Mato Grosso, Rio Grande do Norte, Sergipe, Paraíba, Bahia, Goiás, Minas Gerais, Rio de Janeiro, São Paulo), Bolívia, Paraguai e Argentina.

Plantas-hospedeiras. Bondar (1922) listou quatro espécies da família Arecaceae como plantas-hospedeiras de Coraliomela brunnea brunnea: Cocos nucifera Linn., Syagrus romanzoffiana (Cham.) Glassm., S. schizophylla (Mart.) Glassm. e S. coronata (Mart.) Becc. 
Coraliomela brunnea brunnea: morfologia do adulto e sinonímias...

379
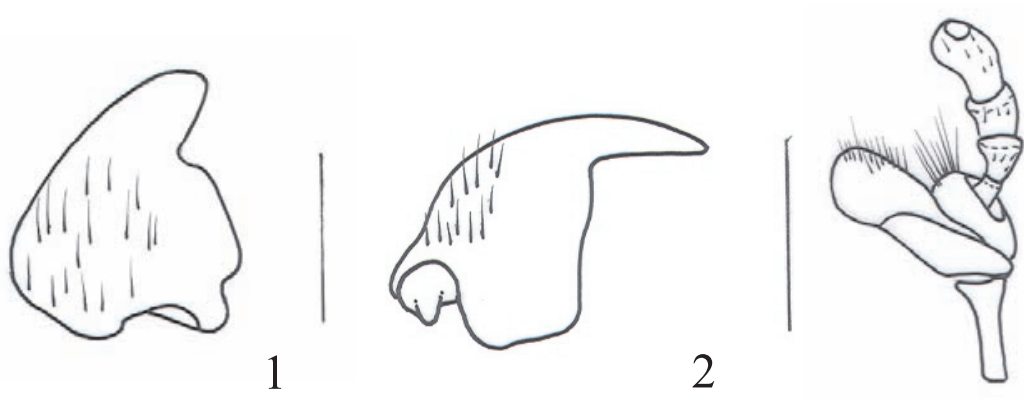

3
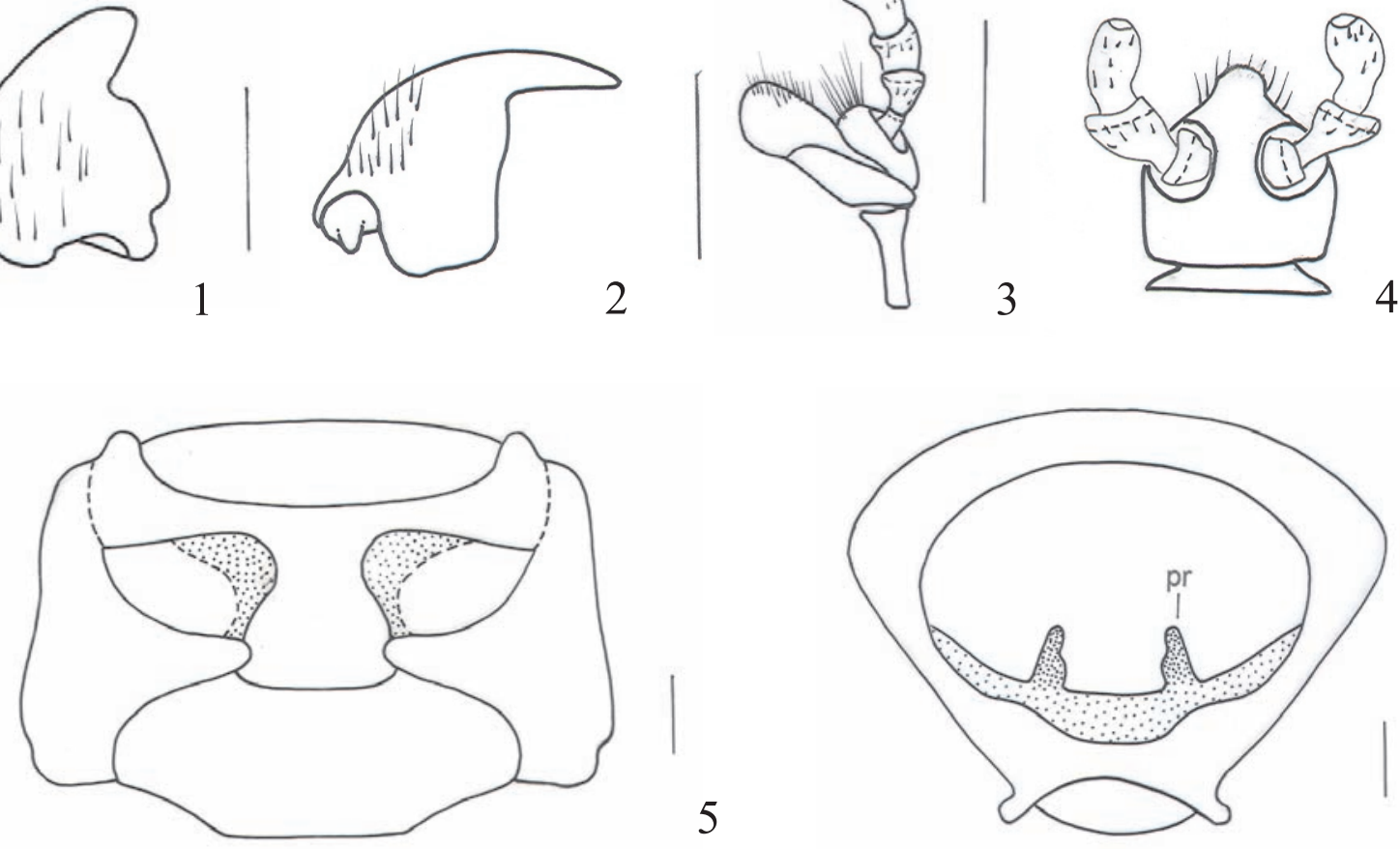

5
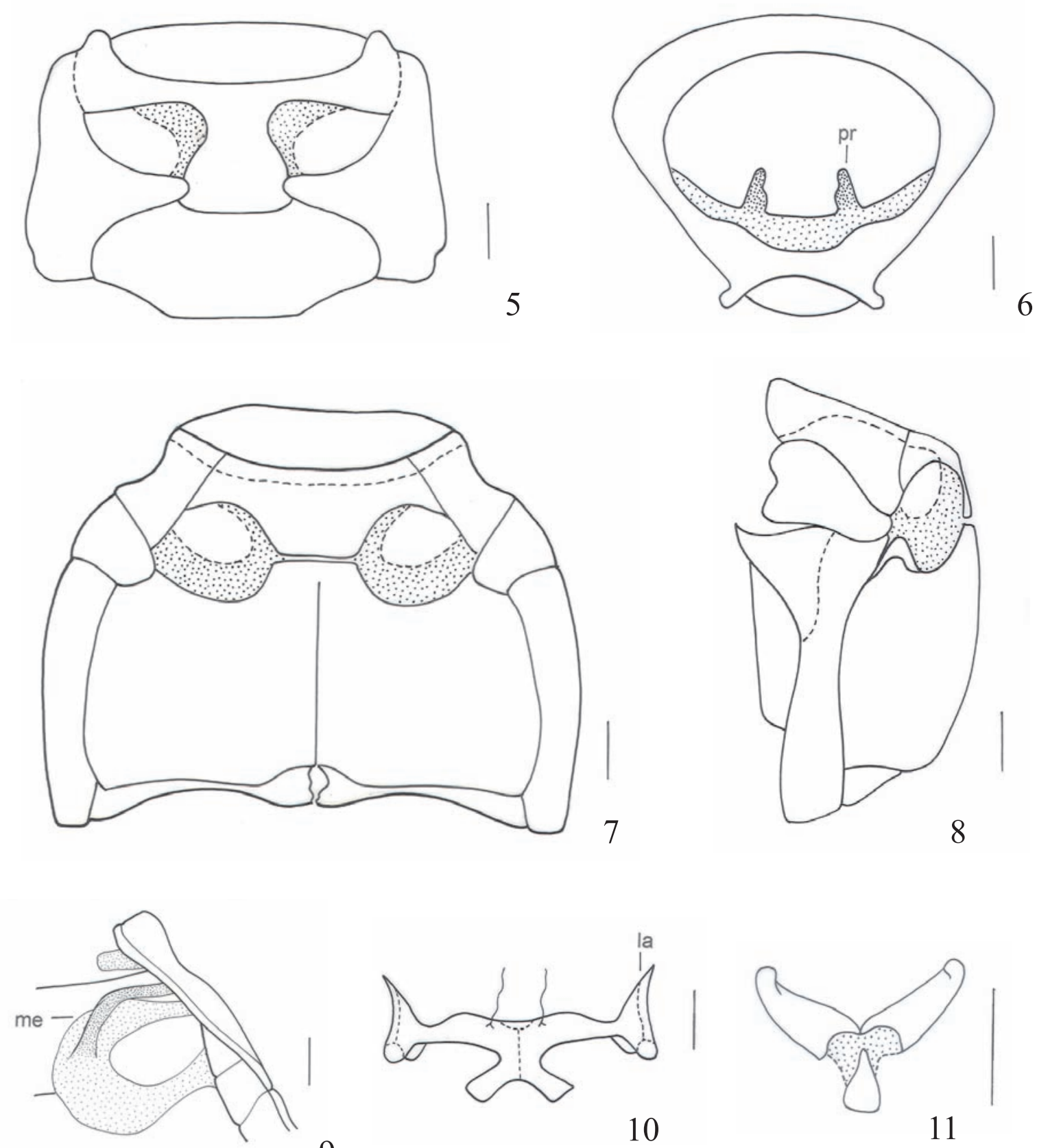

9

10

11

Figs. 1-11. Coraliomela brunnea brunnea (Thunberg, 1821). Mandíbula: 1, dorsal; 2, lateral; 3, maxila; 4, lábio; 5, prosterno; 6, protórax, posterior; 7, mesosterno e metasterno; 8, mesotórax e metatórax, lateral; 9, mesendosternito; 10, metendosternito, dorsal; 11, empódio (la, lâmina; me, mesendosternito; pr, proendosternito). Barras: figs. 1-10, 1,0 mm; fig. 11, 0,5 mm.

Iheringia, Sér. Zool., Porto Alegre, 96(3):377-381, 30 de setembro de 2006 

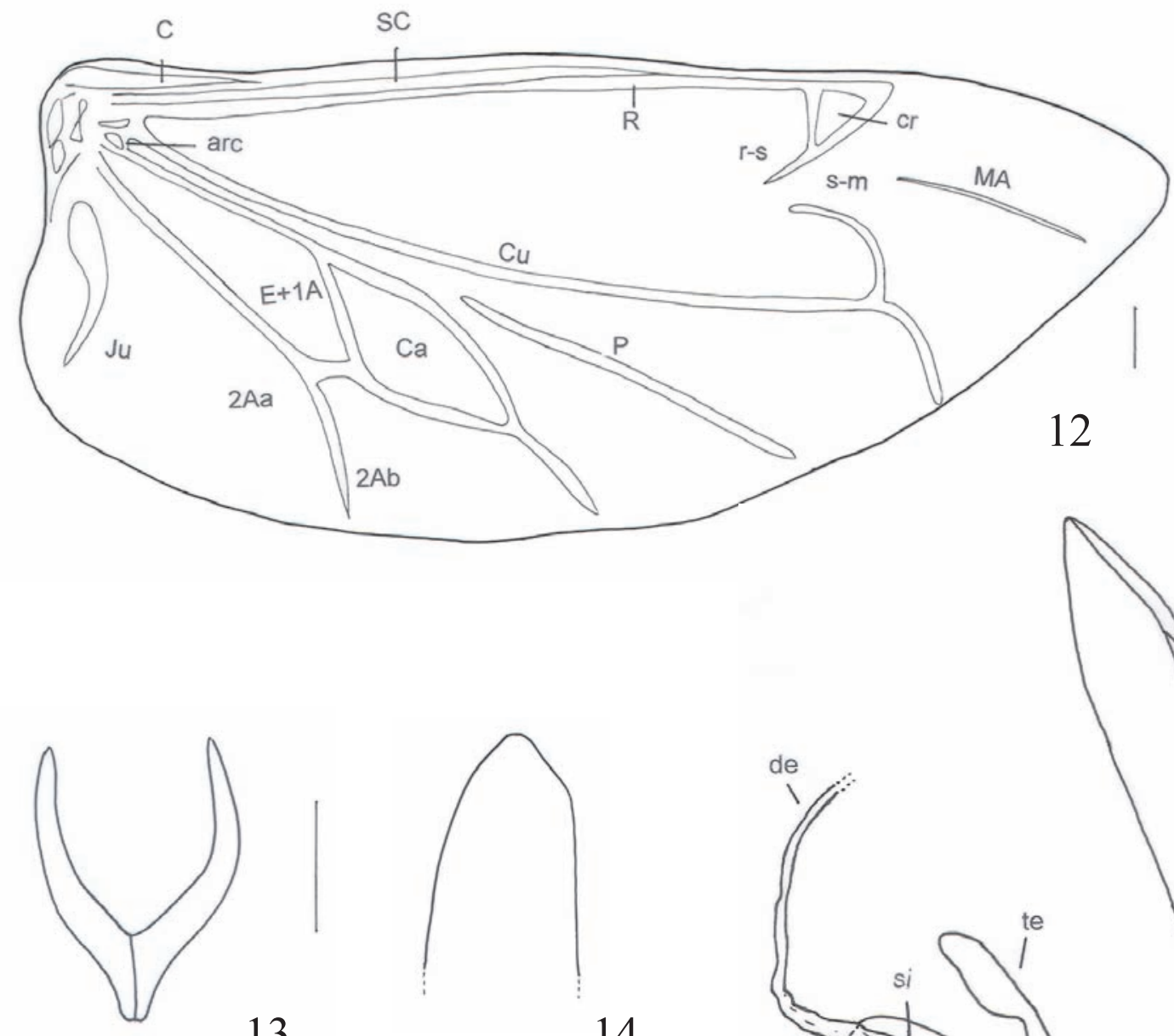

13

14
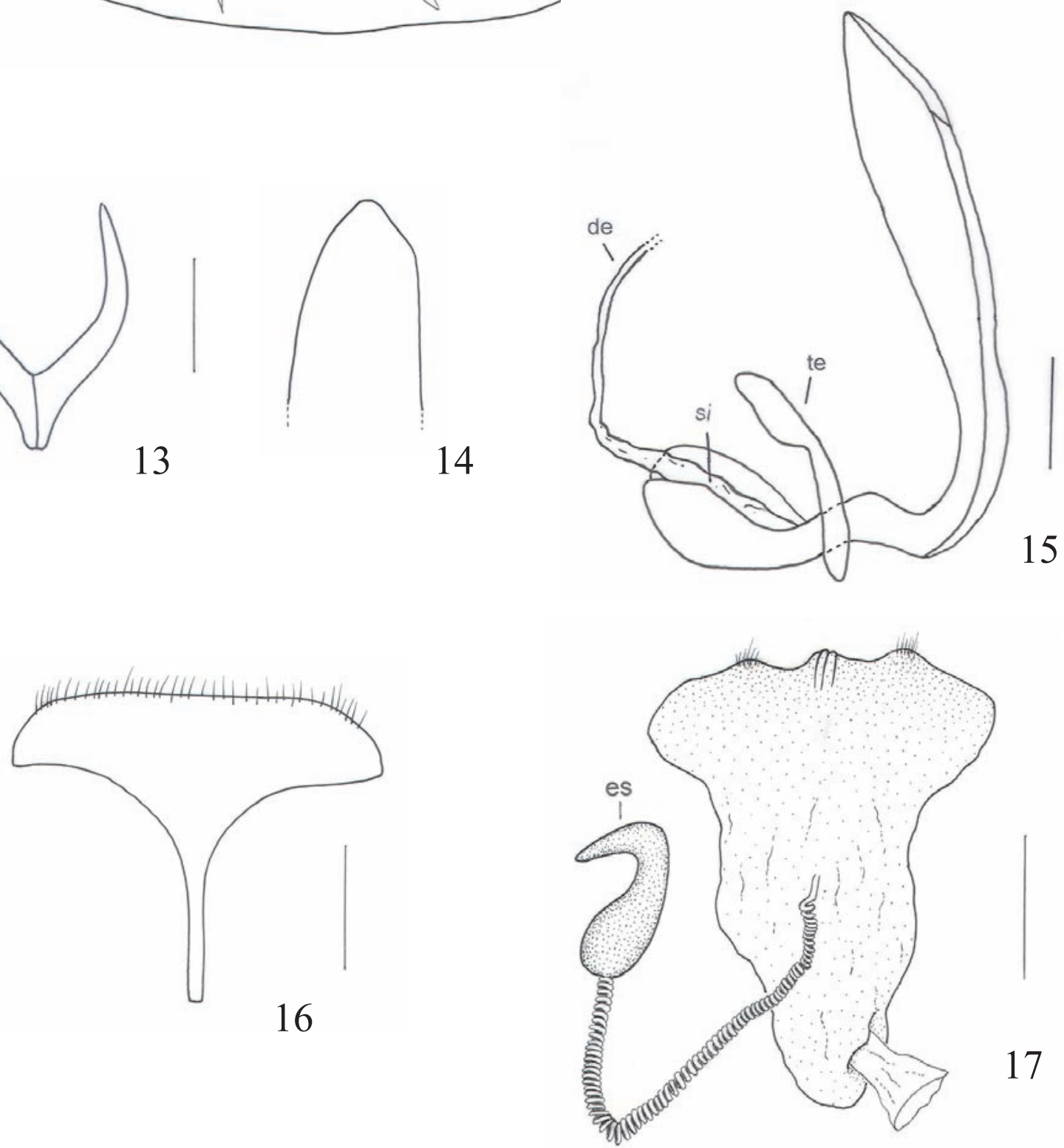

Figs. 12-17. Coraliomela brunnea brunnea (Thunberg, 1821). 12, asa posterior; 13, tégmen, dorsal; 14, lobo médio, ápice; 15, lobo médio e tégmen, lateral; 16, esternito VIII, fêmea; 17, ovipositor (arc, árculo; C, Costa; Ca, Célula anal; cr, célula da radial; Cu, Cubital; de, ducto ejaculatório; E, Empusal; es, espermateca; Ju, Jugal; MA, Média Anterior; P, Plical; R, Radial; r-s, veia transversa rádio setor; SC, Subcosta; si, saco interno; s-m, veia transversa setor média; te, tégmen; $1 \mathrm{~A}, 1^{\circ}$ anal; $2 \mathrm{Aa}$, ramo anterior da $2 \mathrm{~A} ; 2 \mathrm{Ab}$, ramo posterior da 2A). Barras: $1,0 \mathrm{~mm}$. 
Biologia. BONDAR (1922) descreveu a biologia de Coraliomela brunnea brunnea afirmando que a larva vive entre os folíolos dobrados das folhas ou nas axilas de folhas novas do coqueiro; sua presença pode ser percebida pelos estragos causados às folhas e, no final do estágio larval, a larva desce nas axilas de folhas inferiores, onde empupa. Outras informações tratam do ovo, que apresenta comprimento de 7-8 mm, 3-3,5 mm de largura e a duração entre 22-29 dias; a larva, de coloração de "palha morta", com $6 \mathrm{~mm}$ comprimento, $2,5 \mathrm{~mm}$ largura; o período pupal estimado em um mês e o ciclo total aproximadamente de seis meses.

Material examinado. BRASIL, Pará: Belém, 2 exemplares, II.1956, Dirings col. (MZSP); Rondônia: F. P. da Beira, 3 exemplares, 7.XI.1961 F. M. Oliveira col. (DZUP); Mato Grosso: Diamantino, Alto Rio Arinos, 3 exemplares, X.1983, B. Silva col. (MNRJ); Rondonópolis, 2 exemplares, XI.1950, Dirings col. (MZSP); Rosário Oeste, 11 exemplares, 1970, Dirings col. (MZSP); 23 exemplares, I.1972, Dirings col. (MZSP); 5 exemplares, X.1973, Dirings col. (MZSP); Salobra, 1 exemplar, 30.I.1941, F. Lane col. (DZUP); Cáceres, 8 exemplares, XI.1984, C. Elias col. (DZUP); Rio Grande do Norte: Natal, 1 exemplar, 24.X.1951, M. Alvarenga col. (DZUP); 1 exemplar, VIII.1953, Melo col. (MZSP); Paraíba: João Pessoa, 2 exemplares, 5.IV.1953 (MZSP). Bahia: Maracãs, 3 exemplares, 9.XI.1965, F. M. Oliveira col. (DZUP); Goiás: Jataí, 1 exemplar, 1955 (MZSP). Minas Gerais: Campos de Diamantina, 2 exemplares, XII.1902, E. Gounelle col. (MZSP); São Paulo: São Paulo (Ipiranga), 2 exemplares, III.1999 (MZSP); Itu, 1 exemplar, 1.XI.1960, U. R. Martins col. (MZSP); Mogi Mirim (Fazenda Campininha), 5 exemplares, 21.X.1972, M. Sazima e Semir col. (MZSP); Piracicaba, 2 exemplares, XII.1903, Ihering col. (MZSP). BOLÍVIA, Santa Cruz: El Portón, 1 exemplar, 24.IX.1955, P. R. San Martin col. (MNRJ)

Agradecimentos. Ao Luciano de A. Moura (Museu de Ciências Naturais, Porto Alegre), pelas valiosas sugestões ao manuscrito. Aos curadores das instituições, pelo empréstimo do material estudado. À FAPESP pela concessão da bolsa de estudos (Proc. 03/00511-3)

\section{REFERÊNCIAS BIBLIOGRÁFICAS}

BALY, J. S. 1858. Catalogue of Hispidae in the collection of the British Museum. London, Part I, 172p.

BERG, C. 1900. Notice sur les espèces du genre Alurnus F. appartenant à la faune Argentine. Comunicaciones del Museo Nacional de Buenos Aires 1(7):252-257.

Blackwelder, R. E. 1946. Checklist of the coleopterous insects of Mexico, Central America, the West Indies and South America. Part 4. Bulletin of United States National Museum 185:551-763.

BONDAR, G. 1915a. Bichos damninhos da fructicultura e arboricultura. Chacaras e Quintaes 16:21-23

. 1915b. Os coqueiros do littoral brasileiro e suas pragas. Boletim de Agricultura 16:439-441.

1922. Insectos nocivos e molestias do coqueiro (Cocos nucifera) no Brasil. Bahia, Imprensa Official do Estado. p.48-53.

1931. Notas biologicas sobre alguns hispíneos brasileiros. Correio Agricola 9(6): 134-137.

Bose, J. M. 1943. Segunda lista de los Coleópteros de la República Argentina dañinos a la agricultura. Buenos Aires, Ministerio de Agricultura, Dirección de Sanidad Vegetal. 80p.

Bruch, C. 1914. Catálogo sistemático de los Coleópteros de la República Argentina, Parte 9. Revista del Museo de La Plata 19(2):375-376.

Crowson, R. A. 1938. The metendosternite in Coleoptera: a comparative study. Transactions of the Entomological Society of London 87(17):397-416.

1944. Further studies on the metendosternite in Coleoptera.
Transactions of the Entomological Society of London 94(2):273-310

FisCheR, C. R. 1935. Os coleopteros phytophagos da tribu Alurnini, pragas das palmeiras (Chrysomelidae, Hispinae). Revista de Entomologia 5(3):257-292.

Guérin-MénevilLe, F. E. 1840. Note monographique sur le genre de coléoptères nommé Alurnus, etc. Revue de Zoologie 1840:330-332.

JACOBSON, G. 1899. De genere Alurno (Col. Chrysomel.). Annuaire du Musée Zoologique de l'Académie Impériale de Sciences de St. Pétesbourg 4:245-256.

1924. Anotationes synonymicae et systematicae de Colepteris Revue Russe dí Entomologie 18:237-243.

Jolivet, P. 1954. Reserches sur l'aile des Chrysomeloidea (Coleoptera). Deuxième partie. Mémoires de l'Institut Royale des Sciences Naturelles de Belgique 2(58):1-152.

Kasap, H. \& Crowson, R. A. 1985. The studies on the ovipositors and $8^{\text {th }}$ abdominal segments of some species of Bruchidae and Chrysomelidae (Coleoptera). Turkiye Bitki Koruma Dergisi 9(3):131-145.

Lima, A. M. Da Costa. 1923. Catálogo systematico dos insectos que vivem nas plantas do Brasil e ensaio de bibliographia entomologica brasileira. Archivos da Escola Superior de Agricultura e Medicina Veterinária 6(1-2):107-276.

1927. Segundo catálogo systematico dos insectos que vivem nas plantas do Brasil e ensaio de bibliographia entomologica brasileira. Archivos da Escola Superior de Agricultura e Medicina Veterinária 8(1-2):69-301.

1936. Terceiro catálogo systematico dos insectos que vivem nas plantas do Brasil. Rio de Janeiro, Ministério de Agricultura, Escola Nacional de Agronomia 4. 460p.

1955. Insetos do Brasil. $9^{\circ}$ Tomo, Coleópteros, $3^{\text {a }}$ Parte. Rio de Janeiro, Escola Nacional de Agronomia. 289p.

Lindroth, C. H. \& Palmén, E. 1970. Coleoptera, p.80-88. In: Tuxen, S. L. ed. Taxonomist's glossary of genitalia in insects Copenhagen, Munksgaard. 359p.

Mann, J. S. 1985. Studies on the Male Genitalia of Chrysomelidae. III. Galerucinae (Coleoptera: Phytophaga). Annals of Biology 1(1):56-63

Monrós, F. \& Viana, M. J. 1947. Revisión sistemática de los Hispidae Argentinos (Insecta, Coleop. Chrysomeloid.). Anales del Museo Argentino de Ciencias Naturales "Bernadino Rivadavia" 42:125-324.

Moreira, C. 1921. Algumas pragas do coqueiro. Chacaras e Quintaes 23(6):469-471.

1929. Entomologia Agricola Brasileira. Boletim do Instituto de Biologia e Defesa Agrícola 1:79, 82-83.

Ronna, E. 1928. Os insectos do Brasil. São Paulo, Chacaras e Quintaes. $175 \mathrm{p}$.

Spaeth, F. 1937. Ueber die von Regierunsrat E. Reimoser in Argentinien und Paraguay 1907 und 1908 gesammelten Hispinen (Col Chrysom.). Annalen des Naturhistorischen Museum in Wien 48:143-166.

Staines, C. L. 1991. Type species of New World Hispinae genera (Coleoptera: Chrysomelidae). Insecta Mundi 5 (3/4):247-248. 2002. The new world tribes and genera of hispines (Coleoptera: Chrysomelidae: Cassidinae). Proceedings of Entomological Society of Washington 104(3):721-784

TANNER, V. M. 1927. A preliminary study of the genitalia of the female Coleoptera. Transactions of the Entomological Society of London 53:5-50.

ThunBerg, C. P. 1821. Alurni tres novae species descriptae. Nova Acta Regia Societatis Scientiarium Upsaliensis 8:199-202.

VIGORS, N. A. 1826. Descriptions of some rare, interesting, or hitherto uncharacterized subjects of zoology. The Zoological Journal 2:234-242.

Weise, J. 1910. Beitrag zur Kenntnis der amerikanischen Hispinen. Archiv für Naturgeschichte 76(1):67-127.

1911a. In: JunK, W. Coleopterorum Catalogus. Berlin, Pars 35. 94p.

1911b. Coleoptera Phytophaga fam. Chrysomelidae, subfam. Hispinae. In: Wystman, P. Genera Insetorum. Brussels, 125:1-123

Recebido em dezembro de 2005. Aceito em julho de 2006. ISSN 0073-4721

Artigo disponível em: www.scielo.br/isz

Iheringia, Sér. Zool., Porto Alegre, 96(3):377-381, 30 de setembro de 2006 